\title{
Deconvolution of Fluorescence Microscope Imagery
}

\author{
D.S.C. Biggs, *
}

* KB Imaging Solutions LLC, 19 Towpath Ln, Waterford, NY 12188

Deconvolution is a computational technique that can be applied to digital imagery to compensate for the optical limitations of the imaging instrument by reducing out-of-focus blurring or haze. The increased contrast and resolution of the restored data improves not only the visual quality but also the ability to quantify both object dimensions and image intensity. Deconvolution algorithms have been particularly effective in processing 3D fluorescence microscopy data and have been developed into software applications that the general microscope user can routinely use on standard computer hardware.

Unlike astronomical imaging which essentially images a flat 2D scene, in biological microscopy the specimens are typically three-dimensional, requiring multiple optical sections to be captured at different focal positions. With proper Nyquist sampling in both lateral and axial directions a 3D volume of data can be captured that encompasses the whole specimen. However, diffraction in a standard epi-fluorescence microscope limits the smallest lateral resolvable feature to about half the emission wavelength with high numerical-aperture objective lenses, and the axial resolution is even worse. The aberrations inherent in the microscope are modeled by the characteristic point-spreadfunction (PSF) which describes how every point of light emitted by the specimen is observed by the user or camera. The PSF can be easily observed by imaging sub-resolution fluorescence microspheres and focusing through the sample to observe the characteristic hourglass shape.

Mathematically the image observed at the CCD camera is modeled as a convolution between the true 3D light distribution of the specimen and the spatially invariant 3D PSF of the instrument, which is contaminated with Poisson-distributed noise due to photon counting. The ability to restore an accurate representation of the true specimen is limited by the accuracy of the PSF model and the amount of noise contamination. The process to improve the quality of the observed imagery is termed deblurring or deconvolution, depending on the type of algorithm. The convolution operation can be efficiently calculated in the frequency domain using the Fast Fourier Transform (FFT), thus most algorithms make extensive use of FFT's.

One simple deblurring algorithm is the Nearest-Neighbors (NN) method that attempts to remove the blur contribution in the focal plane by subtracting defocused versions of adjacent slices, similar to un-sharp masking. This method requires only 3 optical slices and has low memory and computational requirements; however, the result is only visually qualitative because so much of the image intensity is removed. By contrast deconvolution algorithms attempt to restore the true image intensities and are either linear or iterative (non-linear) in nature.

The inverse or Wiener filter is the typical linear algorithm that can be applied to the whole 3D volume in a single step. There is often a parameter that is adjustable to control the balance between resolution improvement and noise suppression. The Wiener filter can be quickly applied, however, frequencies outside the diffraction bandlimit cannot be restored and there is no inherent limitation on the result having negative intensities values, limiting quantification. 
The most advanced deconvolution algorithms are iterative, requiring multiple cycles to converge towards a desired solution. The iterative algorithms impose non-negativity on the solution, can suppress noise, and even recover frequencies beyond the bandlimit. They come at the cost of increased memory requirements and computational processing. Rather than trying to directly reverse the blurring process, the iterative algorithms estimate the object and use an optimization procedure to improve the result based on the observed data and physical constraints. Typical iterative algorithms are based on measures such as least squares and maximum likelihood (ML). Figure 1 shows the maximum intensity projections of a 3D fluorescence image of spirogyra collected with a 0.75 NA objective lens at $540 \mathrm{~nm}$, and after 10 iterations of a ML-based algorithm.

The quality of the restoration is directly based on the accuracy of the PSF model applied, and estimating an accurate PSF can be difficult. Three typical methods are theoretical calculation using microscope parameters, empirical measurement using sub-resolution beads, and blind or adaptive deconvolution that estimates the PSF directly from the observed data. Each approach has different effects on restoration accuracy, imaging protocol, user effort, and computational requirements.

Once the data has been accurately restored closely based features should be more easily resolvable, object borders more defined, the apparent brightness of the specimen increased, background noise suppressed, and total image intensity preserved. Deconvolution will not make poorly acquired data good, but rather make good data better. In fact, deconvolved data will often show many imaging problems that were previously obscured by the out-of-focus blur. For more in-depth reading see the references [1] - [5].
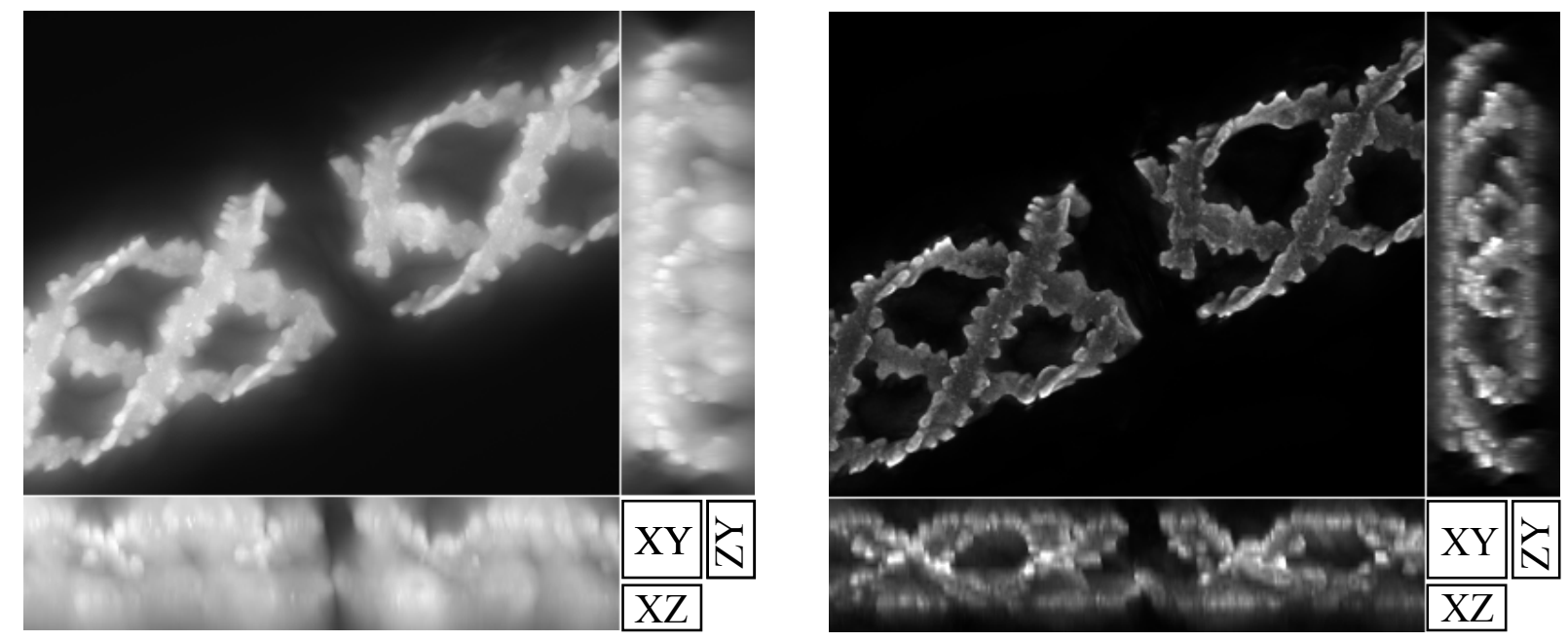

Fig. 1: Maximum intensity projections of original image of spirogyra (left), and after iterative deconvolution (right). XY, XZ and ZY views shown. Volume dimensions (XYZ) are approximately $108 \times 88 \times 25 \mu \mathrm{m}$. Original data courtesy of Olympus-Soft Imaging Solutions (Münster, Germany).

References

[1] D.S.C Biggs, BioPhotonics International, Feb. (2004).

[2] W. Wallace, et al., BioTechniques, Nov. (2001), 1076-1097.

[3] T.J. Holmes, et al., Handbook of Biological Confocal Microscopy, $3^{\text {rd }}$ Ed., Springer, NY, 2006.

[4] J.B. Sibarita, Adv Biochem Engin/Biotechnol, 95: 201-243, Springer-Verlag Berlin 2005.

[5] P. Sarder, et al., IEEE Signal Processing Magazine, Vol. 23, No 3, (2006). 